\title{
Overview of Sulfonamides and Related Medications: Query if Mesalamine should be Preferred Over Dapsone and Sulfasalazine
}

\author{
Craig G. Burkhart ${ }^{*}, 1$ and Craig N. Burkhart ${ }^{2}$ \\ ${ }^{1}$ University of Toledo College of Medicine, USA \\ ${ }^{2}$ University of North Carolina at Chapel Hill, Chapel Hill, North Carolina, USA
}

\begin{abstract}
Sulfonamide research led to the discovery of dapsone and sulfasalzine. Both of these latter drugs have found a niche in the dermatological armamentarium especially for treatment of numerous non-infectious, inflammatory, autoimmune, and bullous disesases. These drugs, however, have side effects which include toxic hepatitis, neuropathy, cholestatic jaundice, hemolysis and methemoglobinemia. In the case of sulfasalamine, it is a conjugate of mesalamine and sulfapyridine. Of note, the latter moiety, sulfapyridine, is no longer available for human use as a separate drug due to side effects. In regards again with sulfasalazine, a pharmacology textbook credits mesalamine to be the major therapeutic moiety, while sulfapyridine is the culprit of the most significant adverse effects.
\end{abstract}

There has been a suggestion in the literature that mesalamine may be the preferred agent for some of these dermatologic therapies for which Dapsone and sulfasalazine are presently suggested. A major impetus to seek this alternate therapy would be its more favorable side effect profile. Certainly more clinical studies using mesalamine are warranted to attest to its clinical effectiveness in relation to the two more established alternatives.

Sulfonamides were developed in the 1930s and were the first effective antimicrobial agent for systemic administration. Sulfonamide research led to the discovery of related compounds, dapsone and sulfasalazine. As a rule, sulfonamides are bacteriostatic (not bacteriocidal), and rely on cellular and humoral host defenses for full eradication of a bacterial infection. Although it now occupies a relatively small place in the therapeutic armamentarium for infections, its usage has recently increased given its benefit in most cases of methicillin-resistant Staphylococcus aureus [1]. Its usage in dermatology may also increase given its effects on the Propionibacterium acnes biofilm [2].

The term 'sulfonamide' refers to chemical structures having the frame composed of para-aminobenzenesulfonamide. Their mechanism of action is competitive antagonist of dihydropteroate synthase, a bacterial enzyme responsible for incorporation of para-aminobenzoic acid for folic acid synthesis. Sulfonamides do not affect human cells by this mechanism, as they require preformed folic acid. Microorganisms which do not synthesize their own folic acid are also resistant to this antibiotic. Trimethoprim is a synergist of sulfonamides because this drug blocks a sequential enzyme in the same pathway by which organisms synthesize folic acid.

Sulfonamides have been used with clinical success in some blistering diseases, but not to the extent of dapsone and sulfasalazine [3].

*Address correspondence to this author at the University of Toledo School of Medicine, 5600 Monroe Street, Suite 106B, Sylvania, OH 43560, USA;

Tel: 419-885-3403; Fax: 419-885-3401; E-mail: cgbakb@aol.com
Dapsone is damino-diphenyl sulfate, which has a chemical structure very similar to sulfonamines. Like sulfonamines, its antibacterial properties are due to inhibiting folic acid synthesis. Although it is most often used in multidrug therapy for leprosy, it has numerous other applications. As an anti-infective agent, it is also utilized in the treatment of malaria and Pneumocystis carinii pneumonia. In dermatology, it has been found helpful in the treatment of numerous non-infectious, inflammatory, autoimmune, and bullous disesases [4]. The list of disorders reported to benefit with dapsone in dermatology include:

- Dermatitis herpetiformis

- Bullous pemphigoid

- Linear immunoglobulin A dermatoses

- Chronic bullous disease of childhood

- $\quad$ Epidermolysis bullosa acquisita

- $\quad$ Pyoderma gangrenosum

- Perifolliculitis capitis abscedens et suffodiens

- Subcorneal pustular dermatosis

- Infantile acropustulosis

- Pustular psoriasis

- Vasculitis

- $\quad$ Erythema elevatum diutinum

- Acne and acne fulminans

- $\quad$ Relapsing polychondritis

- Lupus 
- $\quad$ Behcet's disease

- Granuloma annulare

- Panniculitis

- $\quad$ Lichen planus

- $\quad$ Erythema multiforme

- Mucha Habermann's disease

- $\quad$ Rosacea

- Acrodermatitis continua Hallopeau

- Acro-angiodermatitis.

Prominent side effects of dapsone include toxic hepatitis, cholestatic jaundice hemolysis and methemoglobinemia. Other side effects include nausea, headache, insomnia, psychosis, peripheral neuropathy, coma, and rash. Additionally there is the dapsone hypersensitivity syndrome. This syndrome presents with a triad of fever, skin eruption, and internal organ (lung, liver, neurological and other systems) involvement, occurring several weeks to months after initial administration of the drug [5]. In the PDR, a warning section notes that "Deaths associated with the administration of dapsone have been reported from agranulocytosis, aplastic anemia, and other blood dyscrasias." Of note, the FDA Dermatology Advisory Committee recommends monthly laboratory screening for the first month, then monthly for six months and semi-annually thereafter.

Sulfasalazine (Azulfidine), a chemically related sulfate to dapsone, is poorly absorbed from the gastrointestinal tract, although it fluctuates dependent on gut transit time and gut flora. It is most commonly used for bowel inflammatory disease states. The drug is a conjugate of sulfapyridine and mesalamine (also called 5-aminosalicylic acid). Indeed, this diazo bond is readily cleaved by bacterial azoreductases in the colon releasing these two compounds. Although debated, a pharmacology textbook credits mesalamine to be the major therapeutic moiety, while sulfapyridine is the culprit of the most significant adverse effects [6]. Indeed, sulfapyridine is no longer available by itself for human use as a separate drug. This is related to side effects due to its water solubility with the risk of crystallization within the bladder and urethra.

Although debated, sulfasalazine has been reported to work as well as dapsone in cases of dermatitis herpetiformis [7]. It also has successfully been used in many of the conditions listed above under dapsone including lichen planus, pyoderma gangrenosum, cystic acne, and hidradentis suppurativa $[8,9]$. Moreover, it also has found relevance in the treatment of alopecia areata and atrophie blanche [10-12]. It has been used as a second-line agent in the treatment of arthritis (particularly rheumatoid arthritis), psoriasis and psoriatic arthritis. In the case of arthritis, there is improvement clinically, in laboratory indices, and radiologically [13].

Side effects with sulfasalazine mirrors dapsone including megaloblastic anemia, low sperm counts, agranulocytosis, abnormal liver function, and allergic reactions. Goodman and Gillman's pharmacology textbook suggests that $10-45 \%$ of patients on sulfasalazine experience side effects [6].

Given the potential hematological and urinary side effects, a $\mathrm{CBC}$ with platelet count, liver function, and urinaly- sis are often recommended every 2 weeks for the first 3 months of therapy along with adequate fluid intake [6].

Mesalamine, also referred to as 5-aminosalicylic acid, is an anti-inflammatory agent structurally related to salicylates. It inhibits cyclooxygenase and 5-lipoxygenase pathways of arachidonic acid metabolism. It also acts as a scavenger of reactive oxygen metabolites, affects chemotaxis, phagocytosis, cytokine production and immunoglobulin secretion.

The attachment of mesalamine to sulfapyridine in sulfasalamine prevents early absorption of the aminosalicylate. For higher concentration of the drug in the lower intestines, newer mesalamine preparations allow for higher concentrations to be achieved without the attached sulfonamide by enteric coating, using microgranules with a semipermeable membrane, binding two mesalamine molecules with an azo bond, or linking the product to an inert carrier. These newer aminosalicylic products are generally well tolerated with infrequent side effects.

Of note, administration of unbound or uncoated mesalamine is readily absorbed in the upper jejunum [14-16]. This would be the preferred route for therapeutic purposes of dermatologic disorders. Thus, 5-aminosalicylic acid without agents to block absorption in the upper intestines should be sought for use in skin disorders.

Common side effects with mesalamine include diarrhea, nausea, cramping, and flatulence. Uncommon side effects include headache, hypersensitivity reactions, hair loss, interstitial pneumonitis, and nephritis $[17,18]$.

Unlike dapsone and sulfasalazine, mesalamine has no warning section in the PDR. Also, there is no concern regarding sulfa allergy with this medication. Under precautions, it does suggest a caveat when using the drug with known renal dysfunction or history of renal disease. Additionally, it recommends an evaluation of renal function of all patients prior to initiation of the drug.

There has been the suggestion that mesalamine may be the preferred agent for some of these dermatologic therapies for which dapsone and sulfasalazine are presently suggested [19]. The major impetus would be because of its more favorable side effect profile. Certainly more clinical studies using mesalamine are warranted to attest to, or deny, its clinical effectiveness in relation to the two other alternatives.

\section{REFERENCES}

[1] Genc Y, Ozkanca R, Bekdemir Y. Antimicrobial activity of some sulfonamide derivaties on clinical isolates of Staphylococcus aureus. Ann Clin Microbiol Antimicrob 2008; 7: 7-17.

[2] Burkhart CG. A patient-oriented approach to acne, rosacea, perioral dermatitis, and isotretinoin with working patient informational/instructional sheets and a review of pathophysiology and mechanisms of therapeutic agents. Open Dermatol J 2008; 2: 3643.

[3] Peterson JD, Chan LS. Linear IgA bullous dermatosis responsive to trimethoprim-sulfamethoxazole. Clin Exp Dermatol 2007; 32: 7568.

[4] Wolf R, Orni-Wasserlauf R. A century of synthesis of dapsone: its anti-infective capacity now and then. Int J Dermatol 2001; 39: 779 83.

[5] Kosseifi SG, Guha B, Nassour DN, Chi DS, Krishnaswamy G. The dapsone hypersensitivity syndrome revisited: a potentially fatal multisystem disorder with prominent hepatopulmonary manifestations. J Occup Med Toxicol 2006; 1: 1-9. 
[6] Jafri S, Pasricha PJ. Agents used for diarrhea, constipation, and inflammatory bowel disease: agents used for biliary and parncreatic disease. In: Goodman LS, Gilman A, Hardman JG, Limbird LE, Eds. Goodman and Gilman's. The Pharmacological Basis of Therapeutics. $10^{\text {th }}$ ed. New York: McGraw Hill 2001; pp. 10481050 .

[7] Willsteed E, Lee M, Wong LC, Cooper A. Sulfalazine and dermatitis herpetiformis. Aust J Dermatol 2005; 46: 101-3.

[8] Bauza A, Espana A, Gil P, Lloret P, Vazquez-Dovel J. Successful treatment of lichen planus with sulfasalazine in twenty patients. Int J Dermatol 2005; 44: 158-62.

[9] Shenefelt PD. Pyoderma gangrenosum associated with cystic acne and hidradenitis suppurativa controlled by adding minocycline and sulfasalazine to the treatment regimen. Cutis 1996; 57: 315-9.

[10] Gupta AK, Goldfarb MT, Voorhees JJ. The use of sulfasalazine in atrophie blanche. Int J Dermatol 2007; 29: 663-5.

[11] Aghaei S. An uncontrolled, open label study of sulfasalazine in severe alopecia areata. Indian J Dermatol Venereol Leprol 2008; 74: 611-3.

[12] Rashidi T, Mahd AA. Treatment of persistent alopecia areata with sulfasalazine. Int J Dermatol 2008; 47: 850-2.
[13] Van Rossum MAJ, Fiselier TJW, Franssen MJ, et al. Sulfasalazine in the treatment of juvenile chronic arthritis. Am Coll Rheumatol 1998; 41: 808-16.

[14] Myers B, Evans DN, Rhodes J, Evans BK. Metabolism and urinary excretion of 5-aminosalicylic acid in health volunteers when given intravenously or released for absorption at different sites in the gastrointestinal tract. Gut 1987; 28: 196-200.

[15] Schroeder H, Campbell DES. Absorption, metabolism, and excretion of salicylazosulfapyridine in man. Clin Pharmacol Ther 1972; 13: 539-51

[16] Nielsen $\mathrm{OH}$, Bondesen S. Kinetics of 5-aminosalicylic acid after jejunal instillation in man. Br J Clin Pharmacol 1983; 16: 738-40.

[17] Ransford RA, Langman MJS. Sulphasalazine and mesalazine: serious adverse reactions re-evaluated on the basis of suspected adverse reaction reports to the Committee on Safety of Medicines. Gut 2002; 51: 536-9.

[18] Moss AC, Peppercorn MA. The risks and the benefits of mesalazine as a treatment for ulcerative colitis. Expert Opin Drug Safety 2007; 6: 99-107.

[19] Burkhart CG. Mesalamine may be preferable to sulfasalazine in dermatologic therapeutics. Int J Dermatol 2006; 45: 779-80.

(C) Burkhart and Burkhart; Licensee Bentham Open.

This is an open access article licensed under the terms of the Creative Commons Attribution Non-Commercial License (http://creativecommons.org/licenses/by$\mathrm{nc} / 3.0 /$ ) which permits unrestricted, non-commercial use, distribution and reproduction in any medium, provided the work is properly cited. 